\title{
The influence of emissivity on measured temperature in dependence on spectral range of IR camera detector and its approximate calculation
}

\author{
by J. Tesar*, J. Martan* and J. Skala*
}

\begin{abstract}
* University of West Bohemia, New Technologies - Research Center, Univerzitni 8, Pilsen, Czech Republic, tesar@ntc.zcu.cz, jmartan@ntc.zcu.cz, jskala@ntc.zcu.cz
\end{abstract}

\begin{abstract}
The aim of this article is to show how to get the approximate temperature when the emissivity different from real emissivity of measured surface is used during evaluation. The spectral dependence of measured/calculated temperature is discussed too. In this paper there is presented temperature measurement for three IR cameras of different wavelength ranges on reference blackbody radiators. The influence of emissivity to the evaluated temperatures is made in postprocessing. The simplification from wavelength range to single main wavelength enables direct using of Planck's law to calculation of total radiation flux aiming to the detector. Its analysis with incorrect emissivity leads in resulting approximate temperature.
\end{abstract}

\section{Introduction}

The emissivity is one of the most important properties in quantitative IR thermography. Its knowledge has the crucial influence to the temperature determination accuracy. The influence of emissivity has a spectral dependence too.

\section{Experimental}

Three IR cameras were used for temperature measurements as representatives of detectors in their wavelength ranges - MCS640 (Lumasense) in NIR range (camera detector wavelength range from 780 to $1080 \mathrm{~nm}$ ), SC7650 (FLIR) in MW/SWIR range (1.5 to $5.1 \mu \mathrm{m}$ ) and A615 (FLIR) in LWIR range (7.5 to $14 \mu \mathrm{m})$.

The testing - capturing of single IR image - was made at three different temperatures of blackbody radiators as reference source of defined IR radiation at known temperature. The selected values were $50^{\circ} \mathrm{C}$ as a "low" temperature (near room temperature), $250^{\circ} \mathrm{C}$ as middle temperature and $1000^{\circ} \mathrm{C}$ as high temperature. None of commercial blackbodies has so wide range of temperatures, so three different sources were used - BB701 (Omega) with emissivity value of $\varepsilon=0.95$, BB-4A (Omega) with $\varepsilon=0.99$ and Landcal P1600B2 (Land) with $\varepsilon=0.998$.

The evaluation was made in corresponding software, where the circular analyzed area was placed in the middle of the blackbody cavity as is shown in figure 1. Afterwards the value of emissivity is decreased with constant step and the temperature dependence on emissivity is obtained.
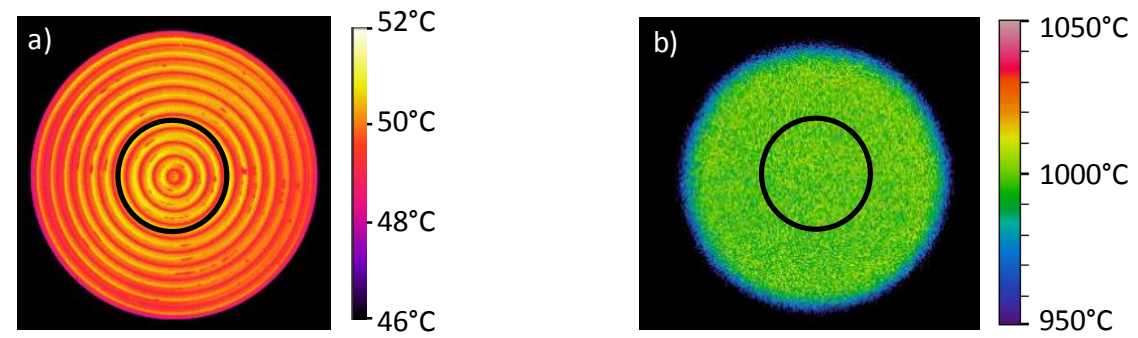

Fig. 1. Thermogram of BB cavity: a) $B B 701, T=50^{\circ} \mathrm{C}$, captured by IR camera FLIR SC7650, b) Landcal P1600B2, $T=1000^{\circ} \mathrm{C}$, captured by MCS640

\section{Theoretical temperature determination}

It would be often very useful to estimate the temperature difference by using incorrect emissivity before the measurement. It is possible with theoretical calculation. It enables to choose proper IR camera device from the view of detector wavelength range and of emissivity determination accuracy.

\subsection{Assumptions of simplification to single main wavelength}

The procedure of temperature calculation is based on basic equation of infrared thermography that states the sum of radiant fluxes absorbed by the detector of measurement device. The sum consists of three terms - the first one corresponds to the radiant flux originated from the surface of measured object, the second one is the radiation heading to 
the detector after reflection from the measured surface and the third is the radiation of atmosphere between measured object and device detector [1] - Eq. (1).

$$
\Phi_{\text {tot }}=\tau_{\text {atm }} \cdot \Phi_{s}+\tau_{\text {atm }} \cdot\left(1-\varepsilon_{s}\right) \cdot \Phi_{r e f l}+\Phi_{a t m}
$$

The parameter $\tau_{\text {atm }}$ characterizes transmissivity of atmosphere and $\varepsilon_{s}$ emissivity of measured object. Emissivity is in the Eq. (1) in the term of reflectivity $\rho_{s}=1-\varepsilon_{s}$ (the measured object is considered as opaque, so $\tau_{s}=0$ ).

The radiant heat flux in Eq. (1) is considered as total (according to Stefan-Boltzmann law $\Phi=\varepsilon \cdot \sigma T^{4}$ ). Used IR camera detectors are active only in ranges of wavelengths mentioned above, so only the parts of the whole spectral range should be calculated. So the correct and exact solution should be to use integration procedure, but the spectral characteristics of optical parameters (emissivity of measured object, transmissivity of atmosphere) and spectral characteristic of detector should have to be used and known. The problem can be simplified to single main wavelength in the first approximation and it enables direct using of Planck's law formula instead of its integration.

\subsection{Principle of temperature calculation}

Consider the situation when the measured object has real emissivity $\varepsilon_{s}$ and the incorrect emissivity $\varepsilon_{d}$ is used to temperature evaluation (set at the detector). Then the incorrect temperature $T_{\mathrm{d}}$ is evaluated and its value can be calculated on the base of equality of total radiant fluxes in the Eq. (1). After simplifying to single main wavelength and introducing emissivities $\varepsilon_{s}$ and $\varepsilon_{d}$ has the equation shape of Eq. (2). The left side of equation represents total radiation intensity with real parameters and right side with parameters set to the detector

$$
\tau_{\text {atm }} \cdot \varepsilon_{s} \cdot L_{s}^{B B}+\tau_{a t m} \cdot\left(1-\varepsilon_{s}\right) \cdot L_{r e f l}^{B B}+\left(1-\tau_{a t m}\right) \cdot L_{a t m}^{B B}=\tau_{a t m} \cdot \varepsilon_{d} \cdot L_{d}^{B B}+\tau_{\text {atm }} \cdot\left(1-\varepsilon_{d}\right) \cdot L_{r e f l}^{B B}+\left(1-\tau_{\text {atm }}\right) \cdot L_{a t m}^{B B}
$$

With using Planck's law the incorrect evaluated temperature can be expressed. All other dependencies to variable parameters can be done by analyzing Eq. (2) (incorrect settings of $T_{\text {refl_d }}-$ temperature of surroundings objects, $T_{\text {atm_d }}$ and $\tau_{\text {atm_d }}$ temperature and transmissivity of atmosphere separately or generally, respectively).

\section{Results}

The evaluated and calculated temperatures for three IR cameras (NIR, MW/SWIR, LWIR) and single main wavelength simplification $(1 \mu \mathrm{m}, 3 \mu \mathrm{m}, 10 \mu \mathrm{m})$ are shown in table 1 . Some of temperatures were not possible to be measured due to too low/high signal (NIR camera starts to measure at $800^{\circ} \mathrm{C}$, MW/SWIR camera has the upper temperature range at $300^{\circ} \mathrm{C}$ ).

Table 1. Results of incorrectly evaluated temperatures when value of $\varepsilon_{d}=0.1$ is set on the detector instead of real object emissivity $\varepsilon_{s}=1$. $\left(T_{\text {refl }}=20^{\circ} \mathrm{C}, \tau_{\text {atm }}=1\right)$. Left side - measured, right side - calculated results, both in ${ }^{\circ} \mathrm{C}$.

\begin{tabular}{|l|c|c|c|c|c|c|c|}
\hline $\begin{array}{l}\text { wavelength } \\
\text { range }\end{array}$ & $\mathrm{T}=50^{\circ} \mathrm{C}$ & $\mathrm{T}=250^{\circ} \mathrm{C}$ & $\mathrm{T}=1000^{\circ} \mathrm{C}$ & $\begin{array}{l}\text { single main } \\
\text { wavelength }\end{array}$ & $\mathrm{T}=50^{\circ} \mathrm{C}$ & $\mathrm{T}=250^{\circ} \mathrm{C}$ & $\mathrm{T}=1000^{\circ} \mathrm{C}$ \\
\hline NIR & out of range & out of range & 1305.4 & $1 \mu \mathrm{m}$ & 67.6 & 297.8 & 1325.8 \\
\hline MW/SWIR & 130.4 & 486.7 & out of range & $3 \mu \mathrm{m}$ & 102.8 & 425.3 & 2627.4 \\
\hline $\mathrm{LWIR}$ & 201.4 & 1211.9 & 5173.9 & $10 \mu \mathrm{m}$ & 202.1 & 1218.3 & 7191.4 \\
\hline
\end{tabular}

Temperatures measured in NIR wavelength range are more independent to the incorrect value of emissivity set at the detector device than in MW/SWIR and in LWIR wavelength range (the higher wavelength, the higher dependency to the value of emissivity).

The measured and calculated values for different temperatures and wavelength ranges/single main wavelengths are in good agreement. Surface temperature calculation based on Planck's law with single main wavelength simplification can be used to the determination of approximate temperature when the incorrect emissivity is set to the device detector.

\section{REFERENCES}

[1] FLIR Systems: User's manual - FLIR A3xx/A6xx sc series, 2012. 